\title{
ON THE POLYNOMIAL INTEGRABILITY OF THE KIRCHOFF'S EQUATIONS
}

\author{
JAUME LLIBRE ${ }^{1}$ AND CLÀUDIA VALLS ${ }^{3}$
}

\begin{abstract}
We prove that the Kirchoff's equations either are completely integrable, or have at most four functionally independent polynomial first integrals.
\end{abstract}

\section{InTRODUCTION AND STATEMENT OF THE MAIN RESUlts}

Given a system of ordinary differential equations depending on parameters in general is very difficult to recognize for which values of the parameters the equations have first integrals because there are no satisfactory methods to answer this question.

In this paper we study the first integrals of the Kirchoff's differential equations in $\mathbb{R}^{6}$ depending on six parameters which provide a model of an ellipsoidal rigid body submerged in an ideal fluid, that is, they are derived under the assumption that the rigid body is ellipsoidal and it is submerged in an infinitely large volume of irrotational, incompressible, inviscid fluid that is at rest at infinity. Under circumstances in which viscous effects are small, it is common to use these equations to describe the dominant dynamics of an underwater vehicle which is ellipsoidal.

The Kirchoff's differential equations appeared by first time in the book of Kirchoff [9]. These differential equations have been studied previously for several authors as Kozlov and Onishchenko [11, 12, 13], Holmes, Jenkins and Leonard [8], see moreover the list of references in these articles about this problem. Many different aspects of the motion of the rigid body governed by the Kirchoff's differential equations where studied in those papers, but the question of its completely integrability using only polynomial first integrals was not considered yet. Here we provide an answer to this question.

The motion of the rigid body has been considered from many other different points of view, for instance many people study the motion of the rigid body fixed at a point, then we get the classical Suslov [14], Chaplygin [3] and Veselov-Veselova [15] problems for the rigid body or generalizations of these problems by Dragovic, Gajic and Jovanovic [6], and many other models non-considered in this work, see for more details on the rigid body the book of Kozlov [10].

Let $p=\left(p_{1}, p_{2}, p_{3}\right)$ and $\pi=\left(\pi_{1}, \pi_{2}, \pi_{3}\right)$ be the linear and angular momentum vectors. Choosing the axes of the body-fixed frame to coincide with the principal axis of the ellipsoid, this yields that the so-called added mass matrix is diagonal, i.e. $M=\operatorname{diag}\left(m_{1}, m_{2}, m_{3}\right)$ and the so-called added inertia matrix is diagonal, i.e. $I=\operatorname{diag}\left(I_{1}, I_{2}, I_{3}\right)$. We recall that each mass and inertia term is the sum of a component due to the body and a component due to the fluid.

2010 Mathematics Subject Classification. Primary 34A05, 34A34, 34C14.

Key words and phrases. Polynomial first integrals, analytic first integrals, Darboux polynomials, homogeneous differential systems. 
Under these conditions, it was proved in [8] that Kirchoff's equations have the form:

$$
\begin{aligned}
\dot{p}_{1} & =\frac{1}{I_{3}} p_{2} \pi_{3}-\frac{1}{I_{2}} p_{3} \pi_{2}, \\
\dot{p}_{2} & =\frac{1}{I_{1}} p_{3} \pi_{1}-\frac{1}{I_{3}} p_{1} \pi_{3}, \\
\dot{p}_{3} & =\frac{1}{I_{2}} p_{1} \pi_{2}-\frac{1}{I_{1}} p_{2} \pi_{1}, \\
\dot{\pi}_{1} & =\left(\frac{1}{I_{3}}-\frac{1}{I_{2}}\right) \pi_{2} \pi_{3}+\left(\frac{1}{m_{3}}-\frac{1}{m_{2}}\right) p_{2} p_{3}, \\
\dot{\pi}_{2} & =\left(\frac{1}{I_{1}}-\frac{1}{I_{3}}\right) \pi_{3} \pi_{1}+\left(\frac{1}{m_{1}}-\frac{1}{m_{3}}\right) p_{3} p_{1}, \\
\dot{\pi}_{3} & =\left(\frac{1}{I_{2}}-\frac{1}{I_{1}}\right) \pi_{1} \pi_{2}+\left(\frac{1}{m_{2}}-\frac{1}{m_{1}}\right) p_{1} p_{2} .
\end{aligned}
$$

They are defined in $\mathbb{R}^{6}$. Note that these differential equations depend on six parameters $I_{i}$ and $m_{i}$ for $i=1,2,3$ and that due to physical constraints they are all positive.

It is well-known (see $[1,2,4,5,8]$ and the references therein) that the Kirchoff equations (1) have always the following three polynomial first integrals of degree two:

$$
H_{1}=\frac{1}{2} \sum_{i=1}^{3} \frac{1}{I_{i}} \pi_{i}^{2}+\frac{1}{2} \sum_{i=1}^{3} \frac{1}{m_{i}} p_{i}^{2}, \quad H_{2}=\sum_{i=1}^{3} p_{i} \pi_{i}, \quad H_{3}=\sum_{i=1}^{3} p_{i}^{2},
$$

which are functionally independent for $i=1,2,3$.

Given $U$ an open set of $\mathbb{R}^{6}$, we say that a real function $H: U \rightarrow \mathbb{R}$ non-constant on any open subset of $U$ is a first integral if it is constant on every solution of system (1) contained in $U$.

The first integrals $H_{1}, \ldots, H_{r}$ are functionally independent if the $r \times 6$ matrix

$$
\left(\begin{array}{ccc}
\partial H_{1} / \partial p_{1} & \cdots & \partial H_{1} / \partial \pi_{3} \\
\vdots & \cdots & \vdots \\
\partial H_{r} / \partial p_{1} & \cdots & \partial H_{r} / \partial \pi_{3}
\end{array}\right)(p, \pi)
$$

has rank $r$ at all points $(p, \pi) \in \mathbb{R}^{6}$ where they are defined with the exception (perhaps) of a zero Lebesgue measure set.

One additional fourth polynomial first integral of degree two functionally independent of the first three is known for the Kirchoff equation if the parameters satisfy the following condition:

$$
m_{1} I_{1}\left(m_{3}-m_{2}\right)+m_{2} I_{2}\left(m_{1}-m_{3}\right)+m_{3} I_{3}\left(m_{2}-m_{1}\right)=0 .
$$

In this case it has the first integral

$$
H_{4}=\left(I_{1}-I_{3}\right)\left(I_{1}-I_{2}\right) p_{1}^{2}+I_{1} I_{3}\left(I_{1}-I_{2}\right) \pi_{2}^{2}+\left(I_{1}-I_{3}\right) I_{1} I_{2} \pi_{3}^{2} .
$$

Here and in what follows to simplify notation we will denote

$$
\alpha_{i}=\frac{1}{I_{i}}, \quad \beta_{i}=\frac{1}{m_{i}}, \quad x_{i}=p_{i}, x_{i+3}=\pi_{i}, \quad i=1,2,3 .
$$


In these new notation we write system (1) as

$$
\begin{aligned}
& \dot{x}_{1}=\alpha_{3} x_{2} x_{6}-\alpha_{2} x_{3} x_{5}=P_{1}\left(x_{1}, x_{2}, x_{3}, x_{4}, x_{5}, x_{6}\right), \\
& \dot{x}_{2}=\alpha_{1} x_{3} x_{4}-\alpha_{3} x_{1} x_{6}=P_{2}\left(x_{1}, x_{2}, x_{3}, x_{4}, x_{5}, x_{6}\right), \\
& \dot{x}_{3}=\alpha_{2} x_{1} x_{5}-\alpha_{1} x_{2} x_{4}=P_{3}\left(x_{1}, x_{2}, x_{3}, x_{4}, x_{5}, x_{6}\right), \\
& \dot{x}_{4}=\left(\alpha_{3}-\alpha_{2}\right) x_{5} x_{6}+\left(\beta_{3}-\beta_{2}\right) x_{2} x_{3}=P_{4}\left(x_{1}, x_{2}, x_{3}, x_{4}, x_{5}, x_{6}\right), \\
& \dot{x}_{5}=\left(\alpha_{1}-\alpha_{3}\right) x_{6} x_{4}+\left(\beta_{1}-\beta_{3}\right) x_{3} x_{1}=P_{5}\left(x_{1}, x_{2}, x_{3}, x_{4}, x_{5}, x_{6}\right), \\
& \dot{x}_{6}=\left(\alpha_{2}-\alpha_{1}\right) x_{4} x_{5}+\left(\beta_{2}-\beta_{1}\right) x_{1} x_{2}=P_{6}\left(x_{1}, x_{2}, x_{3}, x_{4}, x_{5}, x_{6}\right) .
\end{aligned}
$$

We note that condition (2) in this new variables becomes

$$
\alpha_{2} \alpha_{3}\left(\beta_{2}-\beta_{3}\right)+\alpha_{1} \alpha_{2}\left(\beta_{1}-\beta_{2}\right)+\alpha_{1} \alpha_{3}\left(\beta_{3}-\beta_{1}\right)=0 .
$$

By definition the Kirchoff's equations are completely integrable in an open set $U$ if they have 5 first integrals functionally independent in $U$.

Our first result on the integrability of the Kirchoff equations is the next one.

Theorem 1. The Kirchoff's equations satisfying condition (2) are completely integrable.

The proof of Theorem 1 is given in section 2 .

We define the associated vector field $\mathcal{X}$ to the differential system (3) as

$$
\mathcal{X}=\sum_{i=1}^{6} P_{i}\left(x_{1}, x_{2}, x_{3}, x_{4}, x_{5}, x_{6}\right) \frac{\partial}{\partial x_{i}} .
$$

A non-constant polynomial $F \in \mathbb{C}[x]$ is a Darboux polynomial of the vector field $\mathcal{X}$ if there exists a polynomial $K \in \mathbb{C}[x]$ called the cofactor of $F$ such that $\mathcal{X} F=K F$. We say that $F$ is a proper Darboux polynomial if its cofactor is not identically zero, i.e., if $F$ is not a polynomial first integral of $\mathcal{X}$.

Given the involution $\sigma: \mathbb{C}^{6} \rightarrow \mathbb{C}^{6}$ (i.e. a diffeomorphism such that $\sigma^{2}$ is the identity) defined by

$$
\sigma\left(x_{1}, x_{2}, x_{3}, x_{4}, x_{5}, x_{6}\right)=\left(-x_{1},-x_{2}, x_{3},-x_{4},-x_{5}, x_{6}\right)
$$

and a function $F: \mathbb{C}^{6} \rightarrow \mathbb{C}$ we define $F^{\sigma}: \mathbb{C}^{6} \rightarrow \mathbb{C}$ as $F^{\sigma}=\sigma_{*} F=F \circ \sigma$. We say that $F$ is $\sigma$-antisymmetric if $F^{\sigma}=-F$.

Theorem 2. Consider $F$ a proper Darboux polynomial of the vector field $\mathcal{X}$ with cofactor $K$. If $K$ is $\sigma$-antisymmetric then $H=F F^{\sigma}$ is a polynomial first integral of $\mathcal{X}$.

When $m_{1}=m_{2}=m_{3}$, i.e., $\beta_{1}=\beta_{2}=\beta_{3}$ and $I_{1} \neq I_{2}$ and $I_{3} \neq I_{2}$ (i.e. $\alpha_{1} \neq \alpha_{2}$ and $\alpha_{3} \neq \alpha_{2}$ ), then the differential system (3) has the proper Darboux polynomial

$$
x_{4}-\frac{\sqrt{\alpha_{3}-\alpha_{2}}}{\sqrt{\alpha_{2}-\alpha_{1}}} x_{6} \quad \text { with cofactor } \quad K=-\sqrt{\left(\alpha_{3}-\alpha_{2}\right)\left(\alpha_{2}-\alpha_{1}\right)} x_{5} .
$$

Note that $K^{\sigma}=\sigma_{*}(K)=\sqrt{\left(\alpha_{3}-\alpha_{2}\right)\left(\alpha_{2}-\alpha_{1}\right)} x_{5}=-K$ and thus $K$ is $\sigma$-antisymmetric. Then by Theorem 2 system $\mathcal{X}$ has the polynomial first integral

$$
\begin{aligned}
H & =F F^{\sigma}=F \sigma_{*}(F)=-\left(x_{4}-\frac{\sqrt{\alpha_{3}-\alpha_{2}}}{\sqrt{\alpha_{2}-\alpha_{1}}} x_{6}\right)\left(x_{4}+\frac{\sqrt{\alpha_{3}-\alpha_{2}}}{\sqrt{\alpha_{2}-\alpha_{1}}} x_{6}\right) \\
& =-x_{4}^{2}+\frac{\alpha_{3}-\alpha_{2}}{\alpha_{2}-\alpha_{1}} x_{6}^{2} .
\end{aligned}
$$

The proof of Theorem 2 is given in section 3 . 
The main result of this paper goes in the direction of trying to characterize the polynomial first integrals of (3).

Theorem 3. The Kirchoff's equations either satisfy condition (2), or have at most four functionally independent polynomial first integrals.

Theorem 3 is proved in section 4 .

We remark that from the proof of Theorem 3, the result of Theorem 3 can be stated for local analytic first integrals instead of polynomial first integrals.

\section{Proof of Theorem 1}

The following result is due to Jacobi. For a proof in a more general setting see Theorem 2.7 of [7].

Theorem 4. Consider an analytic differential system in $\mathbb{R}^{n}$ of the form

$$
\frac{d x}{d t}=\dot{x}=P(x), \quad x=\left(x_{1}, \ldots, x_{n}\right) \in \mathbb{R}^{n},
$$

with $P(x)=\left(P_{1}(x), \ldots, P_{n}(x)\right)$. Assume that

$$
\sum_{i=1}^{n} \frac{\partial P_{i}}{\partial x_{i}}=0 \quad \text { (i.e. it has zero divergence) }
$$

and that it admits $n-2$ first integrals, $I_{i}(x)=c_{i}$ with $i=1, \ldots, n-2$ functionally independent. These integrals define, up to a relabeling of the variables, an invertible transformation mapping from $\left(x_{1}, \ldots, x_{n}\right)$ to $\left(c_{1}, \ldots, c_{n-2}, x_{n-1}, x_{n}\right)$ given by

$$
y_{i}=I_{i}(x), \quad i=1, \ldots, n-2, \quad y_{n-1}=x_{n-1}, \quad y_{n}=x_{n} .
$$

Let $\Delta$ be the Jacobian of the transformation

$$
\Delta=\operatorname{det}\left(\begin{array}{cccc}
\partial_{x_{1}} I_{1} & \partial_{x_{2}} I_{1} & \cdots & \partial_{x_{n-2}} I_{1} \\
\partial_{x_{1}} I_{2} & \partial_{x_{2}} I_{2} & \cdots & \partial_{x_{n-2}} I_{2} \\
\vdots & \vdots & \ddots & \vdots \\
\partial_{x_{1}} I_{n-2} & \partial_{x_{2}} I_{n-2} & \cdots & \partial_{x_{n-2}} I_{n-2}
\end{array}\right)
$$

Then system (5) admits an extra first integral given by

$$
I_{n-1}=\int \frac{1}{\tilde{\Delta}}\left(\tilde{P}_{n} d x_{n-1}-\tilde{P}_{n-1} d x_{n}\right),
$$

where the tilde denotes the quantities expressed in the variables $\left(c_{1}, \ldots, c_{n-2}, x_{n-1}, x_{n}\right)$. Moreover this first integral is functionally independent with the previous $n-2$ first integrals, that is, the system is completely integrable.

Proof of Theorem 1. It is immediate to verify that the Kirchoff equations (3) in $\mathbb{R}^{6}$ have zero divergence because every $P_{i}$ does not depend on $x_{i}$. In the case of condition (2) the Kirchoff equations have $4=6-2$ first integrals functionally independent. So in this case the Kirchoff equations satisfy the assumptions of Theorem 4. Therefore this case is completely integrable. 


\section{Proof of Theorem 2}

Before proving Theorem 2 we state and prove some auxiliary results and definitions. Given an involution $\tau$, a vector field $\mathcal{X}$ is said to be $\tau$-equivariant if $\tau_{*}(\mathcal{X})=\mathcal{X}$, where $\tau_{*}$ is the push-forward associated to the diffeomorphism $\tau$. We recall that the push-forward $\tau_{*}(\mathcal{X})=(D \tau \mathcal{X}) \circ \tau^{-1}=(D \tau \mathcal{X}) \circ \tau$ because $\tau$ is an involution. Here $D \tau$ denotes the differential of the function $\tau$.

Lemma 5. The vector field $\mathcal{X}$ is $\sigma$-equivariant.

Proof. It follows by direct computations that $D_{\sigma} \mathcal{X}=\mathcal{X} \circ \sigma$.

Lemma 6. If $F$ is a Darboux polynomial of a $\sigma$-equivariant vector field $\mathcal{X}$ with cofactor $K$, then $F^{\sigma}$ is a Darboux polynomial of $\mathcal{X}$ with cofactor $K^{\sigma}$.

Proof. Since $\mathcal{X} F=K F$, we have that $\sigma_{*}(\mathcal{X} F)=\sigma_{*}(K F)$. By the property of pushforwards, we get

$$
\sigma_{*}(\mathcal{X}) \sigma_{*}(F)=\sigma_{*}(K F) .
$$

so $\sigma_{*}(\mathcal{X}) F^{\sigma}=K^{\sigma} F^{\sigma}$. Finally, since $\mathcal{X}$ is $\sigma$-equivariant, $\sigma_{*}(\mathcal{X})=\mathcal{X}$ and we get $\mathcal{X} F^{\sigma}=$ $K^{\sigma} F^{\sigma}$ as we wanted to prove.

Proof of Theorem 2. Under the assumptions of Theorem 2, let $K$ be the cofactor associated to $F$, i.e. $\mathcal{X} F=K F$ with $K \neq 0$. Moreover $K^{\sigma}=-K$, and thus $\mathcal{X} F^{\sigma}=K^{\sigma} F^{\sigma}=-K F^{\sigma}$. Therefore

$$
\mathcal{X} H=\mathcal{X}\left(F F^{\sigma}\right)=(\mathcal{X} F) F^{\sigma}+F\left(\mathcal{X} F^{\sigma}\right)=K F F^{\sigma}+F\left(-K F^{\sigma}\right)=0,
$$

and consequently, $H$ is a polynomial first integral.

\section{Proof of Theorem 3}

We denote by $\mathbb{Z}_{+}$the set of non-negative integers. The following result, due to Zhang [16], will be used in a strong way in the proof of Theorem 3 .

Theorem 7. For an analytic vector field $\mathcal{X}$ defined in a neighborhood of the origin in $\mathbb{R}^{n}$ associated to system (5) with $P(0)=0$, let $\lambda_{1}, \ldots, \lambda_{n}$ be the eigenvalues of $D P(0)$. Set

$$
G=\left\{\left(k_{1}, \ldots, k_{n}\right) \in\left(\mathbb{Z}_{+}\right)^{n}: \sum_{i=1}^{n} k_{i} \lambda_{i}=0, \sum_{i=1}^{n} k_{i}>0\right\} .
$$

Assume that system (5) has $r<n$ functionally independent analytic first integrals $\Phi_{1}(x), \ldots$, $\Phi_{r}(x)$ in a neighborhood of the origin. If the $\mathbb{Z}$-linear space generated by $G$ has dimension $r$, then any nontrivial analytic first integral of system (5) in a neighborhood of the origin is an analytic function of $\Phi_{1}(x), \ldots, \Phi_{r}(x)$.

We call each element $\left(k_{1}, \ldots, k_{n}\right) \in G$ a resonant lattice of the eigenvalues $\lambda_{1}, \ldots, \lambda_{n}$.

Direct calculations show that the Kirchoff equations in (3) have nine planes of singularities, but we only use for proving our result the singularities of the plane $S=\left(x_{1}, 0,0, x_{4}, 0,0\right)$.

At the singularity $S=\left(x_{1}, 0,0, x_{4}, 0,0\right)$, the 6 -tuple of eigenvalues $\mu=\left(\mu_{1}, \ldots, \mu_{6}\right)$ of the linear part of the Kirchoff equations in (3) are

$$
\left(0,0,-\sqrt{\frac{A_{1}-\sqrt{B_{1}}}{2}}, \sqrt{\frac{A_{1}-\sqrt{B_{1}}}{2}},-\sqrt{\frac{A_{1}+\sqrt{B_{1}}}{2}}, \sqrt{\frac{A_{1}+\sqrt{B_{1}}}{2}}\right),
$$


where

$$
\begin{aligned}
& A_{1}=\left(\alpha_{3}\left(\beta_{1}-\beta_{2}\right)+\alpha_{2}\left(\beta_{1}-\beta_{3}\right)\right) x_{1}^{2}-\left(2 \alpha_{1}^{2}+\alpha_{2} \alpha_{3}-\alpha_{1}\left(\alpha_{2}+\alpha_{3}\right)\right) x_{4}^{2}, \\
& B_{1}=-4 \Delta_{1}+A_{1}^{2},
\end{aligned}
$$

with

$$
\Delta_{1}=\left(\alpha_{2}\left(\beta_{1}-\beta_{2}\right) x_{1}^{2}+\alpha_{1}\left(\alpha_{1}-\alpha_{2}\right) x_{4}^{2}\right)\left(\alpha_{3}\left(\beta_{1}-\beta_{3}\right) x_{1}^{2}+\alpha_{1}\left(\alpha_{1}-\alpha_{3}\right) x_{4}^{2}\right) .
$$

From Theorem 7 we know that the number of functionally independent analytic first integrals of the Kirchoff equations in (3) in a neighborhood of the singularities $S_{1}$ is no more than the number of linearly independent elements of the set

$$
G_{1}=\left\{\left(k_{1}, \ldots, k_{n}\right) \in\left(\mathbb{Z}_{+}\right)^{6}: \sum_{i=1}^{6} k_{i} \mu_{i}=0, \sum_{i=1}^{6} k_{i}>0\right\} .
$$

Consequently, the number of the functionally independent polynomial first integrals of the Kirchoff equations in (3) are no more than the number of the linearly independent elements of $G_{1}$.

According to the eigenvalues (6) the resonant lattices satisfy

$$
\sqrt{A_{1}-\sqrt{B_{1}}}\left(k_{3}-k_{4}\right)+\sqrt{A_{1}+\sqrt{B_{1}}}\left(k_{5}-k_{6}\right)=0 .
$$

This last equation has the following linearly independent non-negative solutions $\left(k_{1}, \ldots, k_{6}\right)$ :

$$
(1,0,0,0,0,0),(0,1,0,0,0,0),(0,0,1,1,0,0) \text { and }(0,0,0,0,1,1) \text {. }
$$

In order that equation (7) has other linearly independent non-negative integer solutions different from the above list, we must have

(i) either $\left(A_{1}-\sqrt{B_{1}}\right)\left(A_{1}+\sqrt{B_{1}}\right)=0$;

(ii) or $\left(A_{1}-\sqrt{B_{1}}\right)\left(A_{1}+\sqrt{B_{1}}\right) \neq 0$ and $\sqrt{A_{1}-\sqrt{B_{1}}} / \sqrt{A_{1}+\sqrt{B_{1}}}$ is a rational number. Then $\Delta_{1} \neq 0$ and $A_{1} \neq 0$ (otherwise $\sqrt{-\sqrt{B_{1}}} / \sqrt{\sqrt{B_{1}}}$ cannot be a rational number). Set

$$
\sqrt{A_{1}-\sqrt{B_{1}}} / \sqrt{A_{1}+\sqrt{B_{1}}}=m / n, \quad m, n \in \mathbb{Z} \backslash\{0\} \text { coprime. }
$$

This last equality can be written in an equivalent way as

$$
\frac{\Delta_{1}}{A_{1}^{2}}=\frac{m^{2} n^{2}}{\left(m^{2}+n^{2}\right)^{2}}
$$

where we have used the fact that $B_{1}=A_{1}^{2}-4 \Delta_{1}$.

In case (i) and since $\alpha_{i} \beta_{i}>0$ for $i=1,2,3$, we obtain the following independent conditions:

$$
\begin{array}{lll}
\alpha_{1}=\alpha_{2} & \text { and } & \beta_{1}=\beta_{2}, \\
\alpha_{1}=\alpha_{3} & \text { and } & \beta_{1}=\beta_{3} .
\end{array}
$$

In both cases we are inside the case (4).

In case (ii) $\Delta_{1} / A_{1}^{2}$ has the form $m^{2} n^{2} /\left(n^{2}+m^{2}\right)^{2}$ with $m, n \in \mathbb{Z} \backslash\{0\}$ coprime. So it follows from the expressions of $\Delta_{1}$ and $A_{1}$ that $\Delta_{1}$ should be a square of $\alpha_{2}\left(\beta_{1}-\beta_{2}\right) x_{1}^{2}+$ $\alpha_{1}\left(\alpha_{1}-\alpha_{2}\right) x_{4}^{2}$ or of $\alpha_{3}\left(\beta_{1}-\beta_{3}\right) x_{1}^{2}+\alpha_{1}\left(\alpha_{1}-\alpha_{3}\right) x_{4}^{2}$. Without loss of generality we set

$$
\alpha_{3}\left(\beta_{1}-\beta_{3}\right) x_{1}^{2}+\alpha_{1}\left(\alpha_{1}-\alpha_{3}\right) x_{4}^{2}=L^{2}\left(\alpha_{2}\left(\beta_{1}-\beta_{2}\right) x_{1}^{2}+\alpha_{1}\left(\alpha_{1}-\alpha_{2}\right) x_{4}^{2}\right),
$$

and it is easy to check that $A_{1} /\left(\alpha_{2}\left(\beta_{1}-\beta_{2}\right) x_{1}^{2}+\alpha_{1}\left(\alpha_{1}-\alpha_{2}\right) x_{4}^{2}\right)$ is a constant. Set

$$
A_{1}=K\left(\alpha_{2}\left(\beta_{1}-\beta_{2}\right) x_{1}^{2}+\alpha_{1}\left(\alpha_{1}-\alpha_{2}\right) x_{4}^{2}\right) \text {. }
$$


Then, from (8) and (9) we have

$$
\begin{aligned}
\alpha_{3}\left(\beta_{1}-\beta_{3}\right) & =L^{2} \alpha_{2}\left(\beta_{1}-\beta_{2}\right), \\
\alpha_{1}\left(\alpha_{1}-\alpha_{3}\right) & =L^{2} \alpha_{1}\left(\alpha_{1}-\alpha_{2}\right), \\
\alpha_{3}\left(\beta_{1}-\beta_{2}\right)+\alpha_{2}\left(\beta_{1}-\beta_{3}\right) & =K \alpha_{2}\left(\beta_{1}-\beta_{2}\right), \\
-2 \alpha_{1}^{2}-\alpha_{2} \alpha_{3}+\alpha_{1}\left(\alpha_{2}+\alpha_{3}\right) & =K \alpha_{1}\left(\alpha_{1}-\alpha_{2}\right),
\end{aligned}
$$

where $L / K=m n /\left(n^{2}+m^{2}\right)$. Since $\alpha_{i} \neq 0$ for $i=1,2,3$ we get $\beta_{1}=\beta_{2}=\beta_{3}$ and

$$
\alpha_{1}=\frac{K+2 L^{2} \pm \sqrt{K^{2}-4 L^{2}}}{2\left(1+K+L^{2}\right)} \alpha_{2}, \alpha_{3}=\frac{K+4 L^{2}+K L^{2} \mp \sqrt{K^{2}-4 L^{2}}\left(L^{2}-1\right)}{2\left(1+K+L^{2}\right)} \alpha_{2} .
$$

In all the cases we are inside condition (4).

In short, if cases (i) or (ii) hold then (4) is satisfied and by Theorem 1 the Kirchoff's equations are completely integrable. If cases (i) and (ii) do not hold then by Theorem 7 the Kirchoff's equations can have at most four analytic integrals in a neighborhood of a point of $S$. Consequently the differential system (3) has at most four functionally independent polynomial first integrals. This completes the proof of Theorem 3

Remark 8. By Theorem 7 applied to the singular point $\left(x_{1}, 0,0, x_{4}, 0,0\right)$ the Kirchoff's equations have at most 4 polynomial first integrals. In case that they have exactly 4 polynomial first integrals, the extra analytic first integral provided by Theorem 4 cannot be defined at the singular point $\left(x_{1}, 0,0, x_{4}, 0,0\right)$, otherwise we would have a contradiction with Theorem $\%$.

\section{ACKNOWLEDGEMENTS}

The first author was partially supported by the MICINN/FEDER grant MTM2008 03437, AGAUR grant 2009SGR-410 and ICREA Academia. The second author is supported by AGAUR grant PIV-DGR-2010 and by FCT through CAMGDS, Lisbon.

\section{REFERENCES}

[1] A.M. Bloch, P.S. Krishnaprasad, J.E. Marsden and G. Sáncez De Alvarez, Stabilization of rigid body dynamics by internal and external torques, Automatica 28 (1992), 745-756.

[2] A.M. Bloch and J.E. Marsden, Controlling homoclinic orbits, Theoret. Comput. Fluid Dynamics 1 (1989), 179-190.

[3] S.A. Chaplygin, On rolling of a ball on a horizontal plane (Russian), Collected Papers (Moscow: Nauka), 1976, pp 409-428.

[4] B.D. Coller, P. Holmes And J.L. Lumley, Control of noisy heteroclinc cycles, Physica D 72 (1994), $135-160$.

[5] B.D. Coller And P. Holmes, Supression of bursting, Automatica 33 (1997), 1-11.

[6] V. Dragovic, B. Gajic and B. Jovanovic, Generalizations of classical integrable nonholonomic rigid body systems, J. Phys. A: Math. Gen. 31 (1998) 9861-9869.

[7] A. GoRIELY, Integrability and nonintegrability of dynamical systems, Advanced Series in Nonlinear Dynamics 19, World Sci. Publ. Co., Inc. River Edge, NJ, 2001.

[8] P. Holmes, J. Jenkins And N.E. Leonard, Dynamics of the Kirchoff equations I: Coincident centers of gravity and buoyancy, Physica D 118 (1998), 311-342.

[9] G. Kirchoff, Vorlesungen iiber Mathemahche Physik 1, Mechanik. B, Teubner, Leipzig, 1897.

[10] V.V. Kozlov, Methods of qualitative analysis in the dynamics of a rigid body (Russian), Second edition. Nauchno-Izdatel'skii( Tsentr "Regulyarnaya i Khaoticheskaya Dinamika", Izhevsk, 2000.

[11] V.V. Kozlov and D.A. Onishchenko, Nonintegrability of Kirchhoffs equations, (Russian) Dokl. Akad. Nauk SSSR 266 (1982), 1298-1300. 
[12] V.V. Kozlov And D.A. Onishchenko, On the motion in an ideal fluid of a body containing a moving concentrated mass (Russian), J. Appl. Math. Mech. 67 (2003), 553-564.

[13] V.V. Kozlov And D.A. Onishchenko, Motion of a body with undeformable shell and variable mass geometry in an unbounded perfect fluid, J. Dynam. Differential Equations 15 (2003),553-570.

[14] G.K Suslov, Theoretical mechanics, M.L.: Gostechizdat, 1946.

[15] A.P. Veselov AND L.E. Veselova, Flows on Lie group with nonholonomic constraint and integrable non-Hamiltonian systems, Funct. anal. Appl. 20 (1986), 308-309.

[16] X. ZHANG, Local first integrals for systems of differential equations, J. Phys. A 36 (2003), 12243-12253.

${ }^{1}$ Departament de Matemàtiques, Universitat Autònoma de Barcelona, 08193 Bellaterra, Barcelona, Catalonia, Spain

E-mail address: jllibre@mat.uab.cat

${ }^{3}$ Departamento de Matemática, Instituto Superior Técnico, Universidade Técnica de Lisboa, Av. Rovisco Pais 1049-001, Lisboa, Portugal

E-mail address: cvalls@math.ist.utl.pt 